Revue de l'Institut des langues et cultures

d'Europe, Amérique, Afrique, Asie et Australie

$45 \mid 2022$

Femme(s) et sorcellerie en Espagne et en Italie à l'époque moderne

\title{
Lo strano caso delle streghe di Sicilia: donne de fora, streghe, fate e guaritrici (XVI-XIX sec.)
}

Le cas étrange des sorcières de Sicile: donne de fora, sorcières, fées et guérisseuses (XVI ${ }^{e}-\mathrm{XIX}{ }^{e}$ siècle)

The Strange Case of Sicilian Witches: Witches, Fairies and Healers, from the 16th to the 19th Century

\section{Claudia Geremia}

\section{OpenEdition}

\section{Journals}

Edizione digitale

URL: https://journals.openedition.org/ilcea/14529

DOI: 10.4000/ilcea.14529

ISSN: 2101-0609

\section{Editore}

UGA Éditions/Université Grenoble Alpes

Edizione cartacea

ISBN: 978-2-37747-330-4

ISSN: 1639-6073

Notizia bibliografica digitale

Claudia Geremia, "Lo strano caso delle streghe di Sicilia: donne de fora, streghe, fate e guaritrici

(XVI-XIX sec.) », ILCEA [En ligne], 45 | 2022, mis en ligne le 31 janvier 2022, consulté le 31 janvier 2022 URL : http://journals.openedition.org/ilcea/14529 ; DOI : https://doi.org/10.4000/ilcea.14529

Questo documento è stato generato automaticamente il 31 janvier 2022.

(C) ILCEA 


\title{
Lo strano caso delle streghe di Sicilia: donne de fora, streghe, fate e guaritrici (XVI-XIX sec.)
}

\author{
Le cas étrange des sorcières de Sicile: donne de fora, sorcières, fées et \\ guérisseuses (XVI ${ }^{e}$-XIX ${ }^{e}$ siècle) \\ The Strange Case of Sicilian Witches: Witches, Fairies and Healers, from the 16th \\ to the 19th Century
}

Claudia Geremia

1 Antichissima è l'accusa di stregoneria. Tra le istituzioni che l'hanno formulata, regolamentata e disciplinata, vi è l'Inquisizione, diffusa su un esteso arco cronologico, dal Medioevo all'inizio dell'età contemporanea e su un'area molto vasta: da Malta al Perù, dalla Sicilia a Goa e al Messico coloniale, da Roma a Madrid e Lisbona, dal Cile al Brasile (Prosperi, Lavenia \& Tedeschi, 2010: IV).

2 Nell'Europa cattolica il fenomeno della persecuzione delle donne accusate di stregoneria ebbe il suo apice tra il Quattrocento e il Seicento e fu organizzato e gestito dall'Inquisizione mediante tre tribunali: l'Inquisizione spagnola, quella portoghese e il Sant'Uffizio romano.

3 Il presente saggio ha lo scopo di analizzare alcuni casi delle donne accusate di essere streghe dall'Inquisizione spagnola nel Regno di Sicilia, denominate donne de fora, facendo particolare riferimento alla competenza dei fori riguardante l'accusa di stregoneria. Infatti, come si vedrà in seguito, nel Regno di Sicilia vi era la coesistenza dell'Inquisizione spagnola con quella vescovile, per l'antico privilegio erogato da papa Urbano II nel 1098 nell'Apostolica Legazia (Renda, 2003: 310).

4 Con il termine donne de fora (donne di fuori) si fa riferimento a quelle donne che dicevano di uscire in spirito la notte, lontane dalle loro case per raggiungere raduni stregoneschi organizzati dagli spiriti e da altre streghe. 
Già a partire dalla trattatistica trecentesca compare un primo accenno all'esistenza di alcune donne-fate (de fatis mulieribus) nella Practica inquisitionis heretica pravitatis di Bernard Gui $(1320 \mathrm{ca} \text {.) })^{1}$. Egli Avvertì gli inquisitori circa l'esistenza di queste entità - collocabili tra il naturale e il soprannaturale - e dell'eventualità che escano in spirito la notte (Tedesco, 2020: 71-73).

Il primo studioso che - quasi un secolo dopo l'abolizione dell'Inquisizione spagnola in Sicilia $(1782)^{2}$ - ha riportato alla luce la figura delle donne de fora fu l'antropologo Giuseppe Pitrè. Studiando i documenti conservati nella Biblioteca di Palermo e raccogliendo personalmente informazioni sul campo, tramite le interviste ad alcune persone che dicevano di conoscere le donne de fora, giunse alla conclusione che si trattava di esseri soprannaturali ambivalenti un po'streghe e un po'fate. La caratteristica principale di queste strane donne era la loro ambivalenza; non era possibile riuscire a stabilire se appartenessero al mondo delle fate o a quello delle streghe, né se si trattasse di spiriti o di donne in carne e ossa (Pitrè, 1889: 153).

7 Pitrè, incuriosito dall'essenza di queste donne e soprattutto dalla loro consolidata ambivalenza nella società siciliana, nell'essere riconosciute come un po' fate e un po' streghe, decise di indagare a fondo per scoprirne di più. Dedicò molti mesi alla decodificazione di questo affascinante tema, leggendo alcuni testi di Antonio Veneziano, Vincenzo La Mantia e Antonino Mongitore (eruditi del XVII sec.) e analizzando buona parte dei manoscritti riguardanti il Regno di Sicilia. Intrattenne inoltre un intenso scambio epistolare con eruditi a lui coevi come Raffaele Castelli sul tema delle credenze degli esseri soprannaturali in Sicilia, ma come confessò questi sforzi non lo lasciarono soddisfatto: non riusciva a cogliere l'essenza di queste entità ${ }^{\text {. }}$ Decise quindi di condurre un'ulteriore ricerca su 172 comuni siciliani per capire innanzi tutto se la credenza delle donne de fora fosse radicata su tutto il territorio siciliano o se fosse limitata ad alcune zone della Sicilia e in secondo luogo, il suo intento era quello di capire come venisse recepita dalla popolazione tale credenza. Si rese ben presto conto che le donne de fora erano ancora vive nell'immaginario della popolazione e avevano superato i secoli e la caccia alle streghe. Una differenza in realtà trapelava, riguardava però soltanto la forma delle donne di fora che le sue fonti orali chiamavano con nomi differenti a seconda delle zone della Sicilia: Patruneddi di casa, donne di locu, Belli Signuri (Pitrè, 1889: 163). In alcune zone della Sicilia, come Vizzini (in provincia di Catania) venivano chiamate anche i Lochi di casa poiché preferivano raggiungere direttamente le case per portare la buona o la cattiva sorte (Pitrè, 2020:52). Al di là del nome la sostanza rimaneva sempre la stessa: un po' streghe, un po' fate.

Diversi aneddoti le vedevano protagoniste; si raccontava che le donne de fora si radunassero in una piazzetta sita nel centro storico di Palermo, precisamente nel quartiere Ballarò. A dire di molte persone, sette bellissime donne di notte sceglievano un fortunato da portare con loro in straordinari luoghi. L'incanto finiva con la luce del giorno e le belle fanciulle sparivano. Fu proprio Pitrè a proporre all'ufficio toponomastico il nuovo toponimo "piazza delle sette fate", poiché quel luogo rappresentava, come rilevato dalle sue raccolte, il luogo di incontro di quelle fate che il popolo chiamava donne de fora (Geremia, 2020: 211).

Da quanto è stato detto fin qui le donne de fora, almeno a partire dall'ottocento, sembravano appartenere a un mondo onirico, che poco o nulla aveva a che fare con quello stregonesco. Alla morte di Pitrè rimase irrisolta l'ambivalenza delle donne de fora e per mezzo secolo nessuno studioso si occupò più di sciogliere quello che con lui era 
diventato un enigma, fino a quando Giuseppe Bonomo durante la preparazione del suo libro Caccia alle streghe si imbatté nel caso delle streghe di Sicilia, scorgendone una qualche peculiarità rispetto agli atri casi europei che stava analizzando. Bonomo rimase affascinato dal tema di queste donne-streghe e decise di rintracciare le antiche radici delle magare ${ }^{4}$ di Sicilia, sino a scoprire che quasi un secolo prima dell'insediamento dell'Inquisizione spagnola in Sicilia le donne de fora erano citate nei confessionali (Bonomo, 1971). Questo elemento apriva le porte all'ipotesi di una continuità temporale della credenza delle donne de fora, che l'Inquisizione aveva demonizzato ${ }^{5}$.

Dopo Bonomo, il caso delle streghe di Sicilia fu analizzato da Gustav Henningsen e Carlo Ginzburg. Gustav Henningsen riscoprì le donne de fora presumibilmente studiando alcune delle relaciones de causas, i riassunti annuali che ogni anno i distretti dell'Inquisizione spagnola inviavano alla Suprema. Tra queste carte vi erano i processi inquisitoriali del Regno di Sicilia, celebrati allo Steri di Palermo. Leggendo le vicende delle inquisite e degli inquisiti, trovò un capo d'imputazione ben definito e del tutto peculiare rispetto agli altri distretti inquisitoriali: essere una donna de fora. Così Henningsen rilesse gli studi condotti da Pitrè e Bonomo sul tema e li confrontò con le carte d'archivio, intravedendo la possibilità di una ricostruzione del culto delle fate streghe in Sicilia le cui radici erano antiche e non riconducibili soltanto alla fase inquisitoriale. Ricostruendo il culto delle fate-streghe e pur mantenendo certe riserve si rese conto che l'intuizione che aveva avuto Pitrè nel definire le donne de fora "un po' streghe un po'fate" rappresentasse un punto cruciale di transizione nell'identificazione della figura della fata e poi in quella della strega. Questo passaggio avvenne alla fine del XV secolo in coincidenza con l'istituzione dell'Inquisizione spagnola in Sicilia, infatti prima non esisteva il concetto di demoniaco in Sicilia (Henningsen, 1990: 191-215).

11 In seguito Carlo Ginzburg ha compiuto un passo ulteriore rispetto a Henningsen e ha ipotizzato che la modalità degli incontri notturni delle donne de fora erano molto simili a quelli delle domince nocturnee, seguaci di Erodiade (Diana) e partecipanti del Grande Gioco (Ginzbug, 1989: 101). Infatti nei processi a carico delle donne de fora si fa sempre riferimento sia nella tradizione orale che in quella scritta (processi) a lieti banchetti, danze e incontri gioiosi. L'ipotesi di Ginzburg implicava l'estensione del fenomeno delle donne de fora su un arco cronologico molto esteso, invitando a una riflessione composita sul ruolo svolto dalla macchina inquisitoriale nella trasformazione e demonizzazione di figure come quella in esame.

Alcuni anni dopo Maria Sofia Messana, dedicandosi allo studio della repressione della magia in Sicilia, si imbatté nella strana figura delle donne de fora. Per capire a pieno a cosa fosse dovuta la tanto dibattuta ambivalenza di queste donne, iniziò a ricostruire la categorizzazione degli esseri malvagi fatta dai demonologi tra la fine del Medioevo e la prima età Moderna come Antonio Diana ${ }^{6}$ e Alberto Albertini (Messana, 2007: 212). Oltre all'analisi dei trattati di demonologia, la studiosa riuscì a trovare 77 donne de fora nelle relaciones de causas, rendendosi conto che la maggior parte delle donne inquisite non erano altro che guaritrici (Messana, 2007: 75). Messana elabora, dall'interpretazione delle relaciones de causas, tre modelli interpretativi di donne de fora; questi tre modelli sono il risultato dei tre punti d'osservazione differenti che si hanno nel processo inquisitoriale spagnolo: quello dell'accusatore, dell'imputata e del testimone.

Il primo modello è quello degli inquisitori che sovrappongono la figura delle donne de fora a quelle delle streghe eretiche perseguite in Spagna (brujas), in cui si presuppone 
che alla base dell'eresia vi sia un patto implicito o esplicito con il demonio. Il secondo modello è quello desunto dalla deposizione delle donne accusate di essere donne de fora, le quali raccontano di incontri notturni con altre donne in spirito, di voli e di giardini meravigliosi. Il terzo modello, invece, è quello fornito dai testimoni, i quali parlano delle donne de fora come delle donne in carne e ossa che curano le malattie delle persone e allo stesso tempo riportano quanto raccontatogli dalle imputate. Questi racconti riguardano la dimensione onirica in cui spesso si ritrovano coinvolte le donne de fora, uscendo in spirito la notte (Messana, 2007: 577). Proprio il divario tra la realtà e il sogno, a mio avviso, crea la confusione del definire le donne de fora che sfocia nella dualità strega-fata.

In prospettiva comparativa Julian Goodare ha evidenziato delle somiglianze con il culto scozzese delle seely wights, fate in forma di spirito che la notte solevano vagare e riunirsi con gli altri spiriti alla stessa maniera delle donne de fora (Goodare, 2012: 198-219).

Gli studi di Henningsen, Ginzburg e Messana hanno dimostrato come sia possibile ricostruire specifiche microstorie attraverso i miti e leggende della cultura popolare analizzandoli alla luce dei processi, riassunti dei processi e trattati di demonologia. Da questa analisi emerge una chiara linea del tempo che, almeno per i periodi analizzati, parte dal tardo medioevo e arriva all'età contemporanea. Queste donne de fora vivevano negli agglomerati urbani, non in misteriosi luoghi lontani da tutto e tutti. Si incontrano nel cuore della capitale del Regno di Sicilia, la piazzetta delle sette Fate di Palermo, per celebrare il loro "sabba" che come abbiamo visto consisteva in ricchi banchetti e festose danze. Dall'inizio dei processi inquisitoriali, di cui erano spesse volte le protagoniste, sino ad oggi il mito delle donne de fora è sembrato perdurare e sempre avvolto da quella confusione di cui hanno parlato testimoni, inquisitori e finanche studiosi.

Come precedentemente accennato, l'analisi delle donne de fora è stata fatta tenendo conto dei riassunti dei processi, le relaciones de causas. Da ciò potremmo forse dedurre che se avessimo a disposizione i processi integrali, conosceremmo di più di queste presunte streghe, ma sappiamo che per volontà del Viceré Domenico Caracciolo nel 1783, quasi tutto il materiale relativo all'Inquisizione spagnola in Sicilia è stato bruciato (Sciuti Russi, 2009: 97). E' possibile che altri tipi di fonti, diverse da quelle spagnole, ci rivelino notizie sulle donne de fora?

17 Per tentare di rispondere a questa domanda è necessario tener bene a mente la competenza dei fori in materia di crimini per superstizione nel Regno di Sicilia; vi era infatti la coesistenza del foro ecclesiastico con quello spagnolo. I due fori si occupavano, a volte insieme e a volte autonomamente, della condotta morale dei fedeli per il vecchio principio politico divide et impera (Messana, 2007: 577). I crimini considerati di stregoneria, ma minori, potevano essere giudicati dal foro ecclesiastico, senza tuttavia creare conflitto con la Corte spagnola. La conseguenza di questa coesistenza talvolta produceva una doppia chiamata dinnanzi alle corti, cioè una stessa persona poteva essere convocata per lo stesso reato dai due fori.

Il conflitto di competenza tra i fori giuridicamente non poteva sussistere poiché l'Inquisizione vescovile non fu mai formalizzata e la sua esistenza la si doveva a un antico privilegio che dipendeva dall'estensione e dal potere della contea di Monreale. Anche per questo motivo l'insediamento dell'Inquisizione spagnola in Sicilia non fu né immediato né semplice ma ricco di compressi ed escamotage che garantivano al Regno 
una certa indipendenza. Il più emblematico dei compromessi fu la concessione del privilegio ad alcuni vescovi di diventare inquisitori generali del distretto spagnolo, con pieni poteri spirituali e civici.

Rispetto a quanto detto sopra non è da escludere l'ipotesi che nei processi dell'Inquisizione vescovile si possano trovare interi procedimenti a carico delle donne de fora che contengano più informazioni di quelle ridotte delle relaciones de causas. Questa considerazione è stata l'impulso per iniziare una nuova ricerca sulle donne de fora che partisse proprio dagli archivi diocesani e precisamente da quello di Monreale. Effettivamente nel fondo carte processuali sciolte ho trovato alcuni processi che riguardavano le donne de fora.

Vorrei in questa sede trattare in particolare il processo a carico di Diana La Viscusa, il cui capo d'accusa era essere una donna de fora. Questo processo racconta la storia di quello che potrebbe essere definito un esorcismo, facendo riferimento all'importanza di diversi elementi concorrenti che sembrano essere imprescindibili l'uno dall'altro: la coralità del rito, l'uso di determinati oggetti e il ruolo delle parole nella pratica rituale.

Era il 14 dicembre 1638 quando Ioanni Barrali fu chiamato a deporre dinnanzi al tribunale ecclesiastico della Contea di Monreale contro una certa Diana, nota per essere una delle più potenti streghe del luogo. Ioannis, come riportato dall'inquisitore, appare perplesso e confuso ma consapevole di dover raccontare tutto ciò di cui era a conoscenza per non incorrere in guai ben peggiori di una semplice convocazione dinnanzi al Tribunale. Così Ioannis raccoglie i suoi ricordi, tenuti ben saldi dal terrore che aveva provato nel vedere manifestarsi dinnanzi a lui entità soprannaturali sconosciute e inizia a raccontare la sua storia. Un giorno di luglio del 1638, precisamente il giorno della festa di S. Rosalia (15 luglio) la moglie di Ioannis inizia ad accusare dei forti malori a cui si susseguirono dei deliri come se avesse perso la ragione. Francesca, la moglie di Ioannis, alternava dei momenti di pianto a risate fragorose causate per sua stessa ammissione da strani dolori al cuore e alla testa. Continuò per giorni ad avere questi malori, fino a catturare l'attenzione del vicinato, che aveva suggerito a Ioannis una pratica di uso comune in questi casi, o almeno nel quartiere palermitano del Cassaro, che prevedeva la bastonatura della persona finché non ritornasse normale. Ioannis non era d'accordo a far del male alla moglie, sapeva bene che le implicazioni e le conseguenze di continue bastonature soprattutto sul corpo di una donna potevano essere molto pericolose, così senza pensarci un attimo decise di rivolgersi ad una misteriosa donna di cui aveva sentito molto parlare, nota per le sue doti di guarigione che alcuni chiamavano magara (strega). La strega però non abitava a Cassaro ma a Monreale.

Ioannis continuò la deposizione raccontando che con la moglie raggiunse Monreale e arrivò a casa della strega; Diana ascoltò con molta attenzione tutte le parole di Ioannis. Osservò Francesca e le toccò lo stomaco; poco dopo si premurò di prendere una bacinella piena d'acqua in cui gettare un uovo che aveva iniziato a sbattere. La magara stava praticando l'antica arte delle divinazione per tentare di capire quale male avesse colpito Francesca. L'esito dell'uovo nella bacinella aveva rivelato che si trattava di una magaria; un maleficio aveva colpito Francesca, il costo per guarire la donna era di 6 tarì. Oltre la somma di denaro richiesta, Ioannis doveva acquistare del vino e degli oli che dovevano essere miscelati con dell'acqua e delle erbe in un calderone. Ioannis, come appuntato dall'inquisitore, non ricorda il nome specifico dell'olio né delle erbe. Una volta reperiti i prodotti e consegnati a Diana, lei si premurò di cuocerli sul fuoco; 
l'intento era quello di far respirare a Francesca i suffumigi sprigionati dalle erbe per rimuovere la magaria dal corpo della povera malcapitata. Nonostante il rito, nessun risultato era stato ottenuto e la povera donna continuava a lamentarsi per i forti dolori. Diana così intuisce che il maleficio di Francesca era stato causato per mezzo di oggetto e che doveva necessariamente essere nei pressi o dentro la loro abitazione.

Diana chiese a Ioannis di poter vedere la loro abitazione e tutti insieme andarono a Palermo, precisamente nel quartiere del Cassaro. Diana arrivata dinnanzi all'abitazione iniziò a spargere dell'acqua benedetta e subito dopo scavò una buca all'altezza dell'uscio della porta. Diana, come raccontato da Ioannis all'inquisitore, trovò una carogna di gatto putrefatta con dentro della cera, argento e orina di colore scuro. Quelli erano senza dubbio alcuno gli oggetti del sortilegio, attraverso i quali era stata creato l'incantesimo che arrecava dolore a Francesca.

Diana, rinomata donne de fora, doveva distruggere il sortilegio (magaria) per guarire Francesca. La presunta strega iniziò a bruciare la testa della carogna, cospargendola di palme benedette e rami d'ulivo; la carogna iniziò ad emettere delle strane urla. Ioannis, nel riferire all'inquisitore quanto accaduto, riportò non soltanto il suo stupore ma anche quello dei testimoni, che sentendo delle strane urla accorsero a casa di Francesca e Ioannis. La donna de fora Diana, per proteggere i coniugi e le altre persone che erano sopraggiunte, consegnò a ciascuno di loro una cima d'aruta ${ }^{7}$ che dovevano tenere in mano per proteggersi dai demoni. Così continuò il rito; la presunta strega iniziò a invocare i santi Pietro, Paolo e San Giacomo di Galizia. Francesca iniziò a gridare, dicendo di sentirsi strappare il cuore e le budella. Ad un tratto, iniziò a rimettere dalle parti pubiche "certi cosi nigri, come se fossero zillari" (certe cose nere, come se fossero spilli). Queste "cose nere" erano grumi di capelli di donna infilzati da spilli. A questo punto Diana richiese l'aiuto dei presenti, dovevano ripetere "Signore misericordia" ogni qualvolta che la donna de fora finiva di pronunciare una frase. Bastò questa singola deposizione per far sì che Diana fosse condannata per essere una donna de fora; lei fu condotta al carcere dell'ospedale di Santa Caterina della città di Monreale.

La storia della donna de fora non finisce qui o almeno non in questo modo. Il caso di Diana venne riaperto qualche tempo dopo a seguito delle denunce delle compagne di cella che spaventate richiesero un incontro con l'inquisitore per raccontare ciò che avevano visto.

La detenuta Caterina La Carbonara un giorno ricevette la notizia che il suo amico Bartholomeo era morto a Roma. Diana era presente e disse a Caterina che non era totalmente convinta della morte di Bartholomeo e che se voleva potevano fare un rito per verificare se la notizia fosse vera o no. Caterina disse di sì e Diana iniziò il rito; prese un Rosario e lo divise in tre parti e invocando San Pietro, San Paolo e San Giacomo di Galizia disse le seguenti parole: «se è morto, stai fermo se è vivo muoviti». Secondo quanto riportato dalla testimone, il Rosario iniziò a muoversi da solo. Come ulteriore prova, Diana prese una bacinella, vi orinò dentro e aggiunse degli spilli; mise tutto a cuocere sul fuoco e iniziò a sussurrare alcune parole per non farsi comprendere dai testimoni. Dopo un po' comunicò loro che Bartholomeo fosse vivo.

Dopo la delazione della compagna di cella di Diana, il caso fu riaperto e venne richiamata dall'inquisitore per un nuovo interrogatorio che stavolta prevedeva la tortura. Proprio sotto tortura Diana confessò di aver continuato a praticare l'arte della stregoneria anche in cella; questo gli costò un aggravio della pena. 
30 Acqua benedetta, sale, candele, incenso, rami d'ulivo e palme sono tutti oggetti riconducibili alla sfera cattolica del sacro. Presumibilmente la ragion d'essere dell'uso di questi oggetti cattolici da parte delle presunte streghe, la si trova nella benedizione effettuata dal sacerdote tramite l'utilizzo della parola e quindi questo rientrava nella concezione di sacro. Non a caso gli oggetti precedentemente citati erano allo stesso modo gli strumenti curativi degli esorcisti usati per evocare forze soprannaturali finalizzate alla guarigione. Questi schemi rituali, ripetuti nel tempo, erano stati assimilati come consueti; questo spiegherebbe perché i rituali delle donne de fora non erano intesi come stregoneria da coloro che le frequentavano e ne imploravano l'aiuto. Lo stesso Ioannis, a malincuore depone contro Diana dinnanzi l'inquisitore, perché ne riconosce la capacità di guarire le persone senza porsi troppe domande sulla modalità.

31 Il processo fornisce anche la descrizione completa degli spazi urbani in cui si svolge il rito, prima Monreale e poi Palermo. In secondo luogo, permette anche di concentrarsi sulla funzione corale all'interno della pratica magica svolta da donna de fora. Diana, come abbiamo visto prima, chiese in qualche modo l'aiuto delle persone che assistevano al rito (tramite la ripetizione della frase "Signore misericordia») per amplificarne la forza e allo stesso tempo per proteggersi dai demoni. In questo modo, gli spettatori da quasi inconsapevolmente passivi si trasformavano in partecipanti attivi; proprio in questo momento il rituale si trasforma in un'azione di gruppo. Questa intenzionalità collettiva - che voglio descrivere con la metafora del coro è probabilmente ciò che mancava nelle passate interpretazioni che hanno elaborato dei modelli delle donne de fora.

Vorrei ipotizzare che gli studiosi non siano riusciti a cogliere questo aspetto, perché avevano a loro disposizione solo le relaciones de causas, i riassunti dei processi. I tre modelli di Maria Sofia Messana costituiscono - credo - un tentativo di catturare questa caratteristica dell'azione di gruppo, che ora spero possa diventare ancora più chiara attraverso la lettura di documenti originali. Probabilmente il motivo per cui gli studiosi non sono riusciti a catturare questa intenzionalità collettiva dipende anche dalla mancanza di chiarezza degli stessi osservatori, gli inquisitori. Le diverse parti (testimoni, delatori, imputati, ecc.) hanno menzionato i riti e il nome di chi li ha eseguiti, ciascuno dal proprio punto di vista; questa visione caleidoscopica fu colta a malapena dagli inquisitori al punto da fraintendere il ruolo delle donne de fora. Avere un punto di vista chiaro e unico era essenziale per gli inquisitori, perché dovevano trovare un colpevole e stabilire un crimine. La reazione inquisitoria a questa azione di gruppo sta nella demonizzazione del fenomeno. La demonizzazione non ha cancellato l'inestricabile polarità strega-fata, ma l'ha involontariamente rafforzata e ha contribuito a perpetuarla nel tempo.

$* * * * *$

33 Questo saggio ha cercato di presentare il peculiare caso siciliano delle donne de fora attraverso una breve analisi storiografica e un nuovo caso d'archivio, cercando di fornire una nuova interpretazione grazie all'analisi dei processi integrali del Tribunale di Monreale. Quello analizzato è soltanto uno di altri cinque processi su cui sto lavorando. L'analisi del processo nella versione integrale renderebbe possibile l'eliminazione della mediazione interpretativa che si veniva a creare leggendo solamente le relaciones de causas. La possibilità di approfondire i fenomeni delle donne de 
fora attraverso i processi episcopali rivelerebbe il motivo della complessa confusione sorta nei secoli passati.

Le corti episcopali e spagnole diventano dei contenitori dai quali è possibile estrapolare informazioni riguardanti tradizioni, costumi, credenze e conoscenze terapeutiche della società siciliana di cui le donne de fora ne sono le indiscusse protagoniste. Trattati demonologici, manuali, relaciones de causas, indagini antropologiche e fonti episcopali hanno permesso di tracciare un filo cronologico e tematico che parte dal Medioevo e attraversa l'età contemporanea che resta ancora in parte inesplorato.

BIBLIOGRAFIA

\section{Fonti d'archivio}

Archivio Diocesano di Monreale, fondo carte processuali sciolte, serie 1 busta n. 30,1639.

Archivio Giuseppe Pitrè, Corrispondenti: carpetta P-A-3 n. 90.

\section{Fonti stampate}

DiAna Antonio (1646), In qua A.R.P.D. Antonini Diana Panormitani Clerici Regulis, Venetiis: Iuntas et Baba.

GuI Bernand (1886), Practica inquisitionis haereticae pravitatis, a cura di C. Douais, Paris: Picard.

\section{Fonti secondarie}

ARCidiacono S., NAPOLI M. \& PAVONE P. (1999), Piante selvatiche utilizzate nella medicina e nella veterinaria popolari nel territorio di Bronte (Catania), «Quaderni Botanica, Ambientale Appl.», 10, 105-118.

BоNOMO Giuseppe (1971), Caccia alle streghe. La credenza nelle streghe dal sec. XIII al XIX con particolare riferimento all'Italia, Palermo: Palumbo.

BRANCIFORTI Francesco (1973), Regole, confessionali e rituali, Palermo: Centro di studi filologici e linguistici siciliani.

GEREMIA Claudia (2020), Le donne de fora. Un modello cittadino tra fata e strega nel Regno di Sicilia (secoli $X V$-XIX), E. Ivetic (a cura di), Attraverso la storia. Nuove ricerche sull'età moderna in Italia, Napoli: Editoriale scientifica, 209-219.

GinZBURG Carlo (1989), Storia notturna. Una decifrazione del sabba, Torino: Einaudi.

GOODARE Julian (2012), The Cult of Seely Wights in Scotland, «Folklore», 123, 198-219. 
HENNIngSEn Gustav (1990), 'The Ladies from Outside': An Archaic Pattern of the Witches' Sabbath,

B. Ankarloo \& G. Henningsen (a cura di), Early Modern European Witchcraft: Centres and Peripheries, Oxford: Oxford University Press, 191-215.

MESSANA Maria Sofia (2007), Inquisitori, negromanti e streghe nella Sicilia moderna (1500-1782),

Palermo: Sellerio.

PITRÈ Giuseppe (1889), Esseri soprannaturali e meravigliosi, Id., Usi, costumi, credenze e pregiudizi del popolo siciliano (vol. 4), Palermo: Clio, 153-177.

PITRÈ Giuseppe \& PiRRello Lucia (a cura di) (2020), Streghe siciliane. Malleus Maleficarum siciliensum, Rende: Rossomalpelo.

PROSPERI Adriano (dir.), LAVENIA Vincenzo \& TEDESCHI John (con la collaborazione di) (2010), Dizionario storico dell'Inquisizione, Pisa: Edizioni della Normale.

RENDA Francesco (2003), Storia di Sicilia (vol. 1), Palermo: Sellerio.

SCIUTI RUSSI Vittorio (2009), Inquisizione spagnola e riformismo borbonico fra Sette e Ottocento.

Il dibattito europeo sulla soppressione del «terrible monstre», Roma: Leo S. Olschki.

TEDESCO Vincenzo (2020), Inquisizione, eresia e magia nel tardo Medioevo, Monsagrati: La Vela.

\section{NOTE}

1. Per appronfondimenti, si veda Gui (1886).

2. «Ferdinando IV di Napoli, su richiesta del viceré Domenico Caracciolo, considerando un anacronismo il Sant'Uffizio, ne decretò la soppressione il 16 marzo 1782. L'abolizione fu giustificata con la necessità di restaurare la giustizia, che avrebbe dovuto essere pubblica e fondata sul diritto del regno. Si dispose che il suo patrimonio e le sue rendite fossero incamerate dalla Corona, mentre i suoi pochi ministri e ufficiali furono integrati nel servizio regio. Il dispaccio regio, in realtà, non fece che sanzionare una decisione già presa quattro giorni prima dal viceré, che aveva chiuso e sigillato le dipendenze del tribunale nella sua sede palermitana del Palazzo degli Steri. Era, a giudizio degli illuministi, la fine di una storia di infamia e come tale Caracciolo volle darle il sigillo dell'espiazione e della purificazione, cancellando le testimonianze della vergogna: il 27 giugno $1783 \mathrm{fu}$ ordinato di bruciare gli archivi dell'Inquisizione, riducendo in cenere un pezzo importante della memoria storica siciliana». Si veda Dizionario storico dell'Inquisizione, voce Sicilia, M. Rivero Rodríguez : 1422-1423.

3. Archivio Giuseppe Pitrè, Corrispondenti: carpetta P-A-3 n. 90.

4. Con il termine magara nel dialetto siciliano ci si riferisce alla strega.

5. Per ulteriori approfondimenti sui confessionali medievali che citano le donne de fora, si veda anche Branciforti (1973).

6. «[...] facente brevia cum caracters, seu litteris ignotis, vel recitantes quasdam orazione pro sanandis infermis, fabricantes immagine cerea set puncturis eas cruciando ad amorem vel infirmitatem inducendam, vel maleficia faciendo capillis, ferramentis, clavis, lapibus etc. ab ore maleficiatorum evometis. Item aspicientes speculum aut phialam cum candela, et aqua benedicta, et dicentes aliqua verba pro inveniendis furtis, vel ad scienda secreta» (cit. Summa Diana, 475, c. 69).

7. La cima d'aruta o ruta chalepensis è un'erba della famiglia delle rutacee, veniva spesso usata in Sicilia per la cura dei bambini affetti da ossiuriasi più volgarmente conosciuta come "i vermi"; bastava fare annusare loro un fascio di quest'erba per guarirli. Si veda Arcidiacono, Napoli \& Pavone (1999: 105-118). 


\section{RIASSUNTI}

Il contributo che propongo è incentrato sull'analisi della donna de fora, una particolare figura che compare solo in Sicilia negli atti processuali dei tribunali inquisitoriali spagnoli e vescovili e nella tradizione folklorica. Tale figura femminile è ambivalente: venne considerata dagli inquisitori come sinonimo di brujas (streghe), ma dalle stesse fonti processuali emerge chiaramente come le streghe e i testimoni identifichino nelle donne de fora degli spiriti benevoli assimilabili alle fate, presumibilmente eredi delle domince nocturnee medievali seguaci di Diana. Le protagoniste del mio contributo sono le presunte streghe di Sicilia, raccontate attraverso l'utilizzo di due fonti differenti: i processi inquisitoriali e il materiale etnografico raccolto dall'antropologo Giuseppe Pitrè (1841-1916). Il mio scopo è quello di dimostrare che le fonti di natura diversa (scritte e orali) conducono a un medesimo risultato, permettendoci di delineare una linea di continuità temporale dal XV al XIX secolo.

Ma contribution est centrée sur l'analyse de la donna de fora, une figure particulière qui n'apparaît qu'en Sicile, dans les actes de procès des tribunaux de l'Inquisition espagnols et épiscopaux et dans la tradition folklorique. Cette figure féminine est ambivalente: les inquisiteurs l'ont vue comme l'équivalent de brujas (sorcières), mais il ressort clairement des sources judiciaires que les sorcières et les témoins identifient dans les donne de fora des esprits bienveillants semblables aux fées, dont on peut supposer qu'elles sont les héritières des domince nocturnce médiévales, disciples de Diane. Je présente ici les protagonistes accusées de sorcellerie en Sicile, à travers l'exploitation de deux sources différentes: les procès inquisitoriaux et le matériau ethnographique rassemblé par l'anthropologue Giuseppe Pitrè (1841-1916). Mon but est de montrer que les sources, qu'elles soient écrites ou orales, conduisent à un même résultat nous permettant de tracer une ligne de continuité temporelle du Xv ${ }^{\mathrm{e}}$ au XIX ${ }^{\mathrm{e}}$ siècle.

The "donne de fora (Ladies from outside)" were specific figures that only appeared in the Sicilian trials of the Spanish and Ecclesiastical courts, and in the folkloric tradition. Their ambivalent nature was reflected in the fact that, while they were considered by the inquisitors as true brujas (i.e. witches), both witnesses and the witches themselves saw them as benevolent spirits similar to fairies, descendants of the medieval domince nocturnce followers of Diana. My contribution consists of an analysis of the alleged witches of Sicily. In particular, I will reconstruct their history through the use of two different sources: i) the inquisitorial trials and ii) the ethnographic material collected by the late nineteenth-century anthropologist Giuseppe Pitrè. My aim is to prove how using different types of sources, written and oral, leads to the same findings, outlining a form of temporal continuity from the fifteenth to the nineteenth century.

\section{INDICE}

Parole chiave : donne de fora, Inquisizione spagnola, Regno di Sicilia, streghe, superstizione Mots-clés : donne de fora, Inquisition espagnole, royaume de Sicile, sorcières, superstition Keywords : Ladies from the Outside, Spanish Inquisition, Kingdom of Sicily, witches, superstition 
AUTORE

CLAUDIA GEREMIA

Université de Florence 